Horizons philosophiques

\title{
La science de soi selon Maine de Biran ou le rôle de l'expérience interne
}

\section{Rémy Gagnon}

Volume 10, numéro 1, automne 1999

Écritures et confessions

URI : https://id.erudit.org/iderudit/801111ar

DOI : https://doi.org/10.7202/801111ar

Aller au sommaire du numéro

Éditeur(s)

Collège Édouard-Montpetit

ISSN

1181-9227 (imprimé)

1920-2954 (numérique)

Découvrir la revue

\section{Citer cet article}

Gagnon, R. (1999). La science de soi selon Maine de Biran ou le rôle de l'expérience interne. Horizons philosophiques, 10(1), 129-137.

https://doi.org/10.7202/801111ar d'utilisation que vous pouvez consulter en ligne.

https://apropos.erudit.org/fr/usagers/politique-dutilisation/ 


\section{LA SCIENCE DE SOI SELON MAINE DE BIRAN \\ OU LE RÔLE DE L'EXPÉRIENCE INTERNE}

Mal connue, la doctrine psycho-philosophique de Marie-FrançoisPierre-Gonthier Maine de Biran est probablement celle qui poussera le plus loin l'idée de l'expérience interne comme critère d'appréhension du monde. Au moment où il écrit, la métaphysique cartésienne et le matérialisme physiologique de Cabanis et Destutt de Tracy notamment, l'ont déjà précédé sur cette voie. Ces perspectives influencent sans aucun doute le processus spéculatif d'un pan de la philosophie engagé dans la recherche des causes premières et du principe qui anime les corps vivants. Cabanis par exemple, était parti de lidée que le principe de causalité agit et opère en nous. Puis, il s'était attardé à retracer les mobiles d'action et d'opération de ce principe. Par-delà les avancés de Locke, de Hume et de Condillac (avec qui Maine de Biran entretiendra une polémique) selon lesquelles toutes les idées viennent des sens, Cabanis se demande comment les sensations produisent des idées. Du ressort de la physiologie vitaliste, la réponse qu'il esquisse l'amène à considérer le rapport entre l'organisation physique et les facultés intellectuelles. Le cerveau devenait alors l'agent des impressions, et par là il participait invariablement à toutes émissions de pensée, bref, à toute opération consciente. L'analyse de l'organe cérébral, comme influençant la pensée et la volonté permettait ainsi d'expliquer le rapport entre l'agent de la perception et la chose perçue. Point de départ, mais aussi aboutissement de la réflexion de Maine de Biran, cette forme de sensualisme, comme par ailleurs le cartésianisme, seront des conceptions à renverser. En cela, sa philosophie cherchera à la fois à rompre avec la tradition, et à ouvrir un nouveau chapitre de la réflexion sur le rapport de l'être au monde. Aussi, notre tâche consistera à montrer le potentiel et les limites du projet biranien.

\section{De l'expérience interne comme ouverture sur le monde}

C'est par l'équivoque de la subordination de l'ego à la pure mécanique physiologiste que Maine de Biran en arrive à reposer le problème de la séparation cartésienne entre l'âme substance (qui pense continuellement) 
et le moi qui ne se conçoit que par l'action de celle-ci. En fait, il lui faut être en rupture avec le cartésianisme comme avec le matérialisme physiologique qui finit par affirmer que l'esprit, et que toute conscience, ne s'explique que par le jeu des organes. C'est que la source de l'ego réside, selon Maine de Biran, dans l'immanence, c'est-à-dire dans l'idée d'une âme incorporée au moi qui ne se dévoile que par l'effort. L'âme cartésienne, observatrice du moi s'efface devant une "réalité qui ne sépare pas son être de sa manifestation ${ }^{1} \%$. On pourrait dire que l'existence consciente ne s'aperçoit qu'autant que dure l'effort. En d'autres termes, la critique que Maine de Biran adresse à la métaphysique cartésienne et au matérialisme physiologique, compose l'archétype d'une nouvelle conscience originelle manifestée dans sa dimension d'intentionnalité. "Le sentiment de moi pur si on pouvait le concevoir ainsi par abstraction, nous dit-il, ne serait point (comme pour Descartes) la conscience; la modification ou l'image seule, sans le sentiment de moi, ne l'est pas non plus, car conscience veut dire science (...) science de soi (par rapport à) quelque chose ${ }^{2}$ ». Ainsi posée, la science de l'expérience interne déploie tout son potentiel. Elle seule, croit Maine de Biran, peut affranchir l'homme de ses limites, de l'incompréhension des mécanismes de son être qui le pousse à continuellement attribuer ce qu'il éprouve à des causes extérieures qu'il croit trouver dans les choses. Alors, tout le programme biranien consiste à démontrer linfluence que diverses causes, les unes inhérentes à l'être pensant (ce qui constitue l'essentielle de sa philosophie), les autres extérieures à celui-ci, peuvent exercer sur ce qu'il appelle le fait primitif de la conscience; en d'autres mots, linfluence de l'expérience interne sur la conscience, ou si l'on veut "par quel acte fondamental nous prenons conscience de nous-mêmes et de nos rapports avec l'univers ${ }^{3}$ ".

Le potentiel du programme est immense mais il force, au départ, la substitution de la notion d' «évidence perceptive globale" par une conception plus restreinte et spécifique, fonction de domaines cognitifs particuliers ${ }^{4}$. En fait, cela obligera le philosophe, avant d'identifier la nature ontologique du moi, à cerner les contours du cogito en établissant les

1. Gilbert Romeyer-Dherbey, Maine de Biran, Paris : Seghers, 1974, p. 63.

2. Maine de Biran, "Mémoire sur la décomposition de la pensée", in Oeuvres (éditées par Pierre Tisserand), Paris : Alcan et PUF, 1920-1949, vol. III, p. 69.

3. Raymond Vancourt, La théorie de la connaissance chez Maine de Biran : réalisme biranien et idéalisme, Paris : Aubier, 1947, p. 56.

4. Voir Léon Brunschvicg, L'expérience humaine et la causalité physique, Paris : Félix Alcan, 1922, p. 20. 
limites du perceptif, sinon son domaine précis. Concernant cette nécessaire localisation, il importe de souligner que la réflexion de Maine de Biran s'élabore à partir d'une prémisse essentielle : «les idées ou concepts relatifs à chaque faculté de l'esprit humain ne peuvent s'adresser, en effet, qu'à une faculté de même nature que celle qui les produit5". Cela voudrait dire par exemple, qu'entre la vision d'un géomètre ébahi devant une performance dramatique et son raisonnement qui consiste à demander ce que cela prouve, une incongruité de niveau s'interpose entre le sentiment esthétique et le pragmatisme empirique. Pour paraphraser Maine de Biran, «il ne faut jamais dépasser les limites propres de chaque faculté en voulant appliquer l'une aux objets relatifs à l'autre". Autrement dit, chaque faculté dispose de son propre registre conceptuel, registre qui doit être utilisé comme tel.

L'importance de cette prémisse tient au fait qu'elle met en exergue les dangers de l'empirisme que Maine de Biran cherche à abattre. La conséquence négative de l'évidence empirique, pour qui les hommes sont dominés par l'imagination et les sens, consisterait au fait de rapporter et de mesurer les évidences métaphysiques à l'échelle des sens, même les construits de pur raisonnement. Ainsi, même l'évidence mathématique essentiellement fonction d'un rapport intellectuel, pourrait être concrètement représentée. L'évidence qui se présente au géomètre serait alors médiate : "Elle imprimerait aux figures et au nombre le sceau d'une construction intellectuelle (...) qui repose sur les "longues chaînes de raisons" 6 ", chaînes qui trouvent leur source dans l'ego originaire de la simple expérience subjective.

À cet égard, c'est avec un brin d'ironie que Maine de Biran rend grâce à Hume. «Nul philosophe, écrit-il, n'a établi avec une aussi grande force de conviction qu'il fallait renoncer à trouver hors de nous un fondement réel et solide à l'idée de pouvoir et de force; d'où il suit qu'il n'y a plus qu'à chercher ce fondement en nous mêmes, ou dans le sentiment de notre propre effort, dans le fait même du sens intime dont tous les nuages accumulés par le scepticisme ne sauraient altérer l'évidence" 7 ". Cette évidence qui avait conduit Malebranche, Hume puis Mill à élaborer des doctrines fondées sur un déplacement du sensible vers l'intelligible, sera

5. Maine de Biran, "Essai sur les fondements de la psychologie et sur ses rapports avec l'étude de la nature" [1812], in Ernest Naville ed. Oeuvres inédites de M. Maine de Biran, Paris : Dezorby, E. Magdeleine, 1859, vol. I, p. 134.

6. René Lacroze, Maine de Biran, Paris : PUF, 1970, p. 72.

7. Maine de Biran, "Essai sur les fondements de la psychologie», vol. I, p. 267. 
cependant renversée par Maine de Biran. II s'autorisera à penser que le problème des vérités évidentes en métaphysique, marqué par l'incertitude et la variation, est restreint par un ordre de conscience: la rationalité. Celle-là même qui nous fait appliquer une forme particulière de construit intellectuel, déjà éprouvée entre une sensation et un objet particulier, à toutes les relations expérimentées.

Cet ordre de conscience serait selon Malebranche le point de départ de toute réflexion, il exprimerait la distinction cartésienne entre lidée claire de la substance pensante (forme de conscience) et l'idée claire de la substance étendue, alors que pour Maine de Biran, ce même ordre de conscience serait non pas subséquent et global mais bien antérieur et limité. L'effort volontaire caractérisé par son opposition à la sensation, serait le véritable fait de la conscience. Parce que passive, la sensation subit nécessairement l'influence de l'extérieur, ce qui la rend multiple et variable, alors que l'effort manifesté par le fait de la conscience est au contraire une activité vivante, c'est-à-dire que son opposition au dehors lui révèle son indépendance et suivant, sa permanence. Autrement dit, Maine de Biran, fait de l'effort volontaire, toujours de même nature, l'élément constituant de l'unité du moi où les sensations, les passions et autres faits de conscience viennent se synthétiser. D'abord, "étant donné, écrit-il, une première causalité de fait, nous pouvons en chercher la raison ou l'explication dans la relation des deux substances; mais il serait contraire à toute bonne psychologie de vouloir commencer par l'absolu de la raison, pour en déduire la vérité du fait lui-même ${ }^{8}$ ". Puis il ajoute :

Ce n'est point la liaison des deux substances, ou celle de la force agissante et de son terme d'application immédiate, qui fait le grand mystère de l'humanité ou le grand problème de la psychologie. Cette liaison étant une donnée primitive de la conscience ou de l'existence de l'homme, le mystère serait plutôt dans la possibilité ou la nécessité même de concevoir, ou de croire, la réalité absolue de chacun des termes séparés l'un de l'autre, problème vraiment insoluble a priori, si le fait de conscience ne servait pas d'antécédent et de preuve justificative à la croyance absolue..$^{9}$

8. Maine de Biran, "Exposition de la doctrine philosophique de Leibnitz", in Victor Cousin ed. Nouvelles considérations sur les rapports de physique et du moral de l'homme. Ouvrage posthume de M. Maine de Biran, Paris : Ladrange, 1834, p. 351.

9. Maine de Biran, "Essai sur les fondements de la psychologie», vol. III, p. 440. 
La pensée de Maine de Biran apparaît alors engagée sur la voie de la séparation des substances pensante et étendue, dans la mesure où la substance pensante procède d'une réalité séparée et bien à elle. C'est pourquoi il sera préoccupé de tracer une "ligne de démarcation", afin d'assurer l'originalité et l'indépendance de l'évidence métaphysique du fait autonome de conscience.

Dans la métaphysique, le "concept réfléchi» a pour caractéristique propre «de n'avoir, selon Maine de Biran, aucun signe direct de manifestation; l'évidence du principe est immédiat, tout intérieur, sans que rien d'extérieur puisse la manifester ${ }^{10}$ ". L'évidence psychologique ou métaphysique échappe donc aussi à la forme la plus épurée d'expression par les signes (symboles) : celle véhiculée par les mathématiques. Le problème de cette forme d'expression résiderait dans l'arbitraire des signes qui produisent l'évidence interne sans toutefois la créer, puisque l'évidence psychologique les précède; d'où la nécessaire ligne de démarcation. Ainsi dépourvue de tout symbolisme, l'évidence métaphysique serait plus que jamais éloignée du sensible. Le sens donné au mot je, par exemple, ne suffirait pas, d'abord parce que le signe n'a pas de signification en soi, ensuite parce que «le sens adéquat donné au terme ego ne peut, comme le signale RomeyerDherbey, se faire que par emprunt à la sphère originaire de l'expérience subjective ${ }^{11}$ ». Pour Maine de Biran le signe est symbole tel que l'entendait Aristote, c'est-à-dire comme un repère s'effaçant devant la chose désignée.

Alors, l'ego doit être immanent à l'expérience, il doit être l'expérience pour que le signe soit signifiant. En fait, l'ego de Maine de Biran n'est pas soustrait de la sphère du langage, il en est la source et lui donne son sens. C'est ainsi qu'il déclarera dans une formule lapidaire : "Toute question sur le fait primitif devient frivole, par cela même qu'on en fait une question." Comme Brunschvicg l'a clairement formulé : "L'évidence métaphysique a pour objet propre la réalité intime : c'est à la condition d'éliminer toute intervention, même rationnelle, qu'elle sera en état d'obtenir, ou plutôt de retrouver, le contact avec les faits sous leur forme originelle ${ }^{12}$. Le moi, pourrions-nous ajouter, est ainsi posé comme la cause de tout.

\section{Le causalisme biranien et le fait primitif de la conscience}

De l'empirisme des XVIIe et XVIIIe siècles qui célèbre la primauté des

10. Ibid, vol. I, p. 102-103.

11. G. Romeyer-Dherbey, Maine de Biran, op. cit., p. 77.

12. L. Brunschvicg, op. cit., p. 22. 
sens et soumet, sans amendement possible, la fortune du spiritualisme au dictat de la raison pour ainsi nier la réalité d'une force spirituelle, Maine de Biran retient des considérations d'ordre méthodologique. II s'y autorise, parce que la méthodologie, qui avait permis aux doctrines empiriques d'ériger la sensation en principe véritable et antérieur, lui semble rendre disponibles les armes responsables de sa propre réfutation. En fait, elle lui permet de placer le principe de la conscience biranienne en amont des données sensibles, et cela aux dépens des impressions visuelles, ou plutôt comme Maine de Biran se plaira à le dire, de la etyrannie visuelle ${ }^{13}$ ". Cette opération de désengagement de la conscience par rapport au corps est d'une importance capitale pour la philosophie moderne, dans la mesure où elle fonde une individualité nouvelle dominée par une conscience immanente. En cela, Hume comme Mill auraient donc confondu, ou plutôt simplifié la signification de l'individualité, reconduisant ainsi la méprise de Descartes générée par la formule Je pense donc je suis. En plaçant les deux termes : penser et être dans une formule unique qui, somme toute, ne permet absolument pas de déduire l'un de l'autre, ils auraient en fait subsumé "l'être et l'existence sentie ou aperçue" dans un seul et même terme (absolu), c'est-à-dire qu'à la suite de, et à l'instar de Descartes, ils auraient identifié dans leur esprit l'âme et le moi. ${ }^{14}$ Or, selon Maine de Biran, «(...) la vérité de fait : je pense, et la vérité absolue : je suis une chose pensante, ne sont pas de même genre». La réification cartésienne erre donc en ce qu'elle substantifie le res cogitans dans une formule qui confond le Je pense (vérité déduite) et le Je suis (vérité aperçue). C'est pourquoi Descartes brouille le moi et la conscience du moi. Or, et quoique distincts, ces deux termes ne procèdent, selon Maine de Biran, que d'un rapport à la conscience antérieur à la fois à l'être et à la pensée. Mais alors d'où provient la conscience?

Et bien, la genèse, il la place dans l'acte de volition.

II ne faudrait pas conclure, dit-il, que le fait de conscience est bomé à un seul terme, le sujet absolu (moi). Nous ferons voir, au contraire, qu'il est une véritable dualité, ou un rapport à deux termes de nature homogène. Rien n'est dans la conscience qu'à titre de rapport, et pour qu'un rapport soit dans la conscience, il faut que ses deux ter-

13. En ce qui a trait au problème de la causalité, la vision serait responsable de l'erreur huméenne par excellence qui fait découler l'antécédence et la succession du spectacle que la loi du choc soumet à notre regard.

14. Voir Brunschvicg, op. cit., p. 25-26. 
mes y soient également, sinon comme substance et attribut, du moins comme cause et effet. 15

Ainsi, une distinction fondamentale se fait jour. Dans la philosophie d'inspiration cartésienne le fait primitif de la conscience découle du fait de pensée : c'est elle qui nous donne la certitude de notre existence personnelle. Chez Maine de Biran le fait primitif de la conscience est rendu intelligible suite à l'action libre (la volonté) qui se manifeste et qui se traduit par une résistance organique - un Effort ${ }^{16}$. En fait, la négligence cartésienne du fait primitif de conscience a donné lieu à une interprétation essentiellement axée sur la substance.

Si au lieu de dire vaguement : Je pense donc je suis, Descartes eut dit : Je veux donc je suis, il eût posé d'abord le moi, (comme) cause de ses actes, au lieu d'une âme substance de ses modes, une personnalité, non seulement distincte comme la pensée de l'étendue, mais douée d'une énergie capable de suffire à l'explication des toutes ses opérations et de toutes ses idées. 17

"Nous concevons la substance (mais) nous ne la sentons pas, ajoute Maine de Biran, nous ne l'apercevons pas intimement, tandis que nous apercevons en nous la force, en même temps que nous la concevons hors de nous ou dans l'objet ${ }^{18}$ ». Provocateur s'il en est un, cet énoncé suppose un ordre de conscience de l'être au monde à la fois antérieur et postérieur à celui-là. Antérieur parce que la conscience qui préexiste est activée par l'effort, et postérieur parce que la conscience une fois activée permet la reconnaissance et l'exploration du monde. C'est pourquoi concluait-il que «ces philosophes (de l'École de Descartes) confondant la notion commune que nous avons de la cause efficiente dont le type est notre moi, avec la notion mystique d'une cause créatrice des substances, ont cru que notre raison pouvait atteindre celle-ci sans passer par la première ${ }^{19}$ ". Ainsi, entre la notion de substance et la notion de causalité il y a une différence radicale de rythme: suivant l'une on va du dehors au dedans, suivant l'autre du dedans au dehors. L'aperception de conscience donne la connaissance du moi grâce à l'activité irréductible de la volonté comme action immanente à l'effort, car il n'y a pas d'effort involontaire. Si bien que la théorie de Maine

15. Maine de Biran, "Essai sur les fondements de la psychologie», vol. I, p. 152.

16. Voir Vancourt, La théorie de la connaissance, op. cit., p. 61-66.

17. Victor Cousin, "Préface de l'éditeur", in Victor Cousin ed. Nouvelles considérations, op. cit., p. xviii.

18. Maine de Biran, "Exposition de la doctrine philosophique de Leibnitz", p. 329.

19. Citée par A. Drevet, Maine de Biran : L'effort, Paris : P.U.F., 1966, p. 129. 
de Biran ne reconnaît que ces trois axiomes : 1) la vraie activité est dans la volonté; 2) la volonté c'est la personnalité, le moi lui-même; 3) vouloir c'est causer, et le moi est la première cause qui nous est donnée. L'acte de volition qui conduit à un effort est attesté par la conscience, et le rapport qui les lie en est un de causalité. La volition, comme fait primitif évident par lui-même, trouve ainsi sa place au fond de l'effort, et agit non seulement à titre de cause, mais bien à titre de cause volontaire. ${ }^{20}$ Maine de Biran vise, par là, à révolutionner la philosophie de la recherche des causes pour la faire s'intéresser au fait le plus réel d'entre tous : l'existence du moi (tel qu'il l'entend). ${ }^{21}$ Victor Cousin résume parfaitement sa philosophie :

Dans la continuité de l'effort, le moi se sent toujours vouloir et agir, et il se sent la même volonté et la même cause, alors même que les effets voulus et produits varient. Ce moi identique et un, distinct de ses effets variables, ne tombe ni sous les sens ni sous l'imagination; il s'aperçoit lui-même directement dans la continuité de son activité qui est pour lui la continuité même de son existence; il existe donc incontestablement pour lui-même d'une existence qui échappe à l'imagination et aux sens : c'est là l'existence spirituelle.22

C'est donc en s'abattant sur le sensualisme avec l'arme redoutable de l'être comme cause véritable et première, par opposition aux sensations qui sont extérieures et donc l'effet de l'action du moi, que Maine de Biran a pu prétendre renouveler non seulement la conception de la causalité, mais également l'histoire entière des systèmes philosophiques. En cela, il préfigure sans doute les Husserl, Heidegger et Gadamer.

Mais bien sûr, il n'est pas une seule philosophie qui puisse entièrement satisfaire l'esprit humain, et celle de Maine de Biran n'échappe pas à cette règle. Ce philosophe de l'être qui propose une version de la conscience ouverte sur le monde par le biais de la volonté, qui place l'acte de volition en amont des idées, ce philosophe dis-je, ne peut se soustraire à l'aporie ultime que pose la question de l'origine des idées purement intellectuelles. Comment en effet, faire découler les idées de l'éternel et de l'infini de l'acte de la volonté bref, imaginer que celles-ci puissent être causées par la

20. Voir Philip P. Hallie, Maine de Biran Reformer of Empiricism 1766-1824, Cambridge, Harvard University Press, 1959, pp. 84-104.

21. On le voit, la tentative de s'élever jusqu'à la cause des causes se rattache directement à la monadologie de Leibnitz. Et Maine de Biran le confessera dans son Exposition de la doctrine philosophique de Leibnitz initialement rédigée pour la Biographie Universelle de Michaud.

22. Cousin, "Préface de l'éditeur", op. cit., p. xi. 
simple volonté? Chez Maine de Biran, cette question demeure sans réponse, sinon lorsqu'il esquisse, à l'instar de Descartes et Newton, le plan d'action d'une force déifiée. Car pour peu que l'on accepte l'expérience interne comme condition de la présence des idées, et suivant de la conscience, il faut néanmoins reconnaître qu'une condition n'est pas une cause de la conception de l'éternité et de l'infinitude propre à l'Homme, dans la mesure où la nature même de ces idées les fait se poser au-delà de l'existence du moi. Aussi faut-il voir dans la philosophie biranienne du moi cause de tout, une tentative de passage à vide vers les deux principes constitutifs de la nature humaine : "une vie inconsciente ayant ses lois spéciales étrangères à toute intervention de la volonté, et une vie proprement humaine dont la conscience est le caractère et dont la volonté est l'agent ${ }^{23}$ ". Mais cette tentative, Maine de Biran ne la conçoit nettement qu'à la fin de sa vie, au moment où la tâche du philosophe se perd dans le dédale des incertitudes de l'esprit.

Rémy Gagnon

Faculté de théologie, d'éthique et de philosophie Université de Sherbrooke

23. Ernest Naville, Maine de Biran, sa vie et ses pensées, Paris : Joël Cherbuliez, 1857, p. 109. 